\title{
Near miss audit in obstetrics
}

\author{
Robert Pattinson, \\ MRC Maternal and Infant Health Care Strategies Research Unit, \\ University of Pretoria, \\ Gauteng, South Africa \\ * Tel.: +27 12373 1002; Fax: +27 123731045
}

Confidential enquiries into maternal deaths (CEMD) have long been a valuable tool for assessing the quality of care in pregnancy. CEMDs have the advantage of a clear reference point. For practical purposes defining a maternal death is simple and unambiguous.

In developed countries the Maternal Mortality Ratio’s (MMR) are low. To get a large enough sample to identify trends in the quality of maternal health care or of the diseases causing maternal deaths takes anything from 3 to 10 years. The information might be out of date due to new developments and discoveries by the time the information is published. Further, the circumstances related to a maternal death in a developed country are often very particular to the individual case and the value of this information for general use might be limited.

In countries where maternal deaths are relatively frequent, CEMDs give a good indication of problems in the health system, provided most deaths occur within health institutions. Maternal deaths per institution however are still uncommon, even in countries with a high MMR. An area has to be large to gather reliable information on problems within the health system if maternal deaths are to be used as the audit tool. Thus to use audits of maternal death as a tool to evaluate the quality of maternal care a large reporting structure needs to be created and sustained. This structure can be very effective in helping develop the country's health care strategy. However, it's relevance to a smaller area might be limited.

Collecting information on all maternal deaths in an area is more difficult, increasingly so when the numbers of births at home increase. Understanding the factors surrounding these home deaths becomes more important as the proportion of deaths occurring outside of institutions rises. Analysis on institutional deaths might give a misleading picture if the home deaths are ignored. Collecting information on home deaths is labour intensive, expensive and dependent on verbal autopsies. Often home deaths can only be assessed during periodic studies, and cannot be used as a routine audit tool.

This edition of Best Practice provides an effective method of evaluating the quality of care using near misses, both maternal and neonatal. Analysis of maternal near misses gives a mechanism of supplementing information gained from CEMDs and in some cases replacing them.

The big stumbling block to use maternal near misses as a reliable audit tool has always been the criteria used to define a near miss. Any definition of criteria must take into account all countries (low, middle and high income) and must not be dependent on the resources available in that country. The chapter by Say et al., gives such criteria. They have been validated for their use in defining maternal near miss and these criteria can be used in all countries. This is a major break through making auditing of maternal near miss an activity to assess the quality of maternal care that can be readily undertaken by health institutions, districts, regions and even countries. Examples of these are given in the chapters on the use of maternal near miss in low and high income countries. The rapidity of finding useful information from near miss audits is another advantage over CEMDs.

The use of maternal near miss audits in not restricted to problems within the health institutions. The chapters by Ronsmans and Filippi et al., both clearly illustrate their value in low income settings. Maternal near miss analysis might also be able to overcome the problems related to the use verbal autopsies in quality of care issues and clearly are very useful in determining the factors involved in the three delays that are so often involved in maternal deaths.

Maternal near miss analysis helps keep us from forgetting previous lessons that taught us how to reduce the maternal deaths in an area. The chapters on managing the unconscious pregnant woman, acute collapse of the pregnant women and postpartum haemorrhage are examples of not forgetting these valuable lessons. Maternal and or neonatal near misses also often lead to long term complications and the chapter by Lang describes this well.

The basis of the concept of maternal near miss can also be extended into evaluating the quality of intrapartum care by identifying neonatal near misses. This concept is developed by Avenant and a provides a good example of criteria that can be used for a neonatal near miss. The chapter on hypoxic ischaemic encephalopathy by Buchmann and Velaphi takes what is clearly a neonatal near miss and illustrates how this can be usefully used to evaluate the quality of intrapartum care.

Near miss audit in obstetrics is a concept that can now be adopted as a valuable tool for auditing maternal care. Its time has come. 